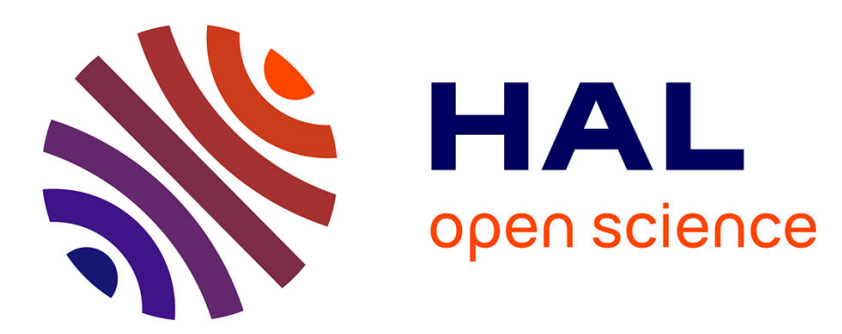

\title{
Stability analysis and estimate of the region of attraction of a human respiratory model
}

Rafael Villafuerte, Sabine Mondié, Silviu-Iulian Niculescu

\section{To cite this version:}

Rafael Villafuerte, Sabine Mondié, Silviu-Iulian Niculescu. Stability analysis and estimate of the region of attraction of a human respiratory model. IMA Journal of Mathematical Control and Information, 2010, 27 (4), pp.493-511. 10.1093/imamci/dnq033 . hal-00557970

\section{HAL Id: hal-00557970 \\ https://hal.science/hal-00557970}

Submitted on 17 Jul 2019

HAL is a multi-disciplinary open access archive for the deposit and dissemination of scientific research documents, whether they are published or not. The documents may come from teaching and research institutions in France or abroad, or from public or private research centers.
L'archive ouverte pluridisciplinaire HAL, est destinée au dépôt et à la diffusion de documents scientifiques de niveau recherche, publiés ou non, émanant des établissements d'enseignement et de recherche français ou étrangers, des laboratoires publics ou privés. 


\title{
Stability Analysis and Estimate of the Region of Attraction of a Human Respiratory Model
}

\author{
Raul Villafuerte*, Sabine Mondié*, and Silviu-Iulian Niculescu ${ }^{\dagger}$ \\ *Department of Automatic Control CINVESTAV-IPN, A.P. 14-740, México D.F., \\ MÉXICO. e-mail: \{rvillafuerte, smondie\}@ctrl.cinvestav.mx. \\ ${ }^{\dagger}$ Laboratoire des Signaux et Systèmes (L2S), CNRS-Supélec, rue Joliot Curie, \\ 91192, Gif-sur-Yvette, France. e-mail:Silviu.Niculescu@lss.supelec.fr
}

\begin{abstract}
In this paper, we complete the stability analysis of various human respiratory nonlinear time delay models introduced in (Vielle \& Chavet 1998), (Kollár \& Turi 2004), (Batzel \& Tran 2000a), and (Batzel \& Tran 2000b). More precisely, we present a detailed mathematical analysis of the stability of the nonlinear model trivial equilibrium, an estimate of its region of attraction and exponential estimates of the solutions starting in this region. The proposed approach is constructive and it is based on the use of Lyapunov-Krasovskii functionals of complete type for time-delay systems with a cross term in the time derivative.
\end{abstract}

\section{Introduction}

Roughly speaking, the respiratory system includes two compartments, lungs and lumped body tissue connected by the circulating blood (Timischl 1998), (Hoppensteadt \& Peskin 1992) and it is characterized by the presence of two types of processes: the distribution of $\mathrm{O}_{2}$ to the cells and the elimination of the $\mathrm{CO}_{2}$ in the tissues of the body (Murray 1993). The classical way to represent the dynamics of such compartments defining the respiratory system is based on the mass balance equations. For example, the change of $\mathrm{CO}_{2}$ volume in the lung compartment is determined by the balance between the expired/diffusion rate of $\mathrm{CO}_{2}$ expired from/into the lungs. A similar mass balance equation holds also for the change of $\mathrm{O}_{2}$ (see, e.g., (Timischl 1998)). In the absence of voluntary control of breathing or neurological induced changes in breathing, the physiological human respiratory control system varies the ventilation rate in response to the levels of $\mathrm{CO}_{2}$ and $\mathrm{O}_{2}$. In this context, 
without any attempt for a deeper discussion on the modelling part, the breathing process in the biological circuit controlling the carbon-dioxide level in the blood is a transport process that is typically represented by a set of delay differential equations.

The aim of this paper is to propose a deeper analysis of the stability properties of the trivial solution of different proposals of nonlinear models of the human respiratory system with transport delays in their representation that are encountered in the literature. More precisely, we will consider four models: (Vielle \& Chavet 1998), (Kollár \& Turi 2004), (Batzel \& Tran 2000b), and (Batzel \& Tran 2000a). In our analysis, we will exploit explicitly the particular structure of the system and the properties of the system's nonlinearities. Furthermore, the analysis is completed by providing an estimate of the region of attraction of the trivial solution and an exponential estimate of the solutions whose initial conditions are in this region. To the best of the authors' knowledge, there does not exist similar results and comparisons for the analysis of the respiration system. The approach considered here is based on the use Lyapunov-Krasovskii functionals of complete type (Kharitonov \& Zhabko 2003) with crossing term in the time derivative (Mondié, Kharitonov \& Santos 2005). Such an approach is inspired by the ideas introduced in (Melchor-Aguilar \& Niculescu 2007). It should be mentioned that the estimate of the domain of attraction has also been successfully studied in the framework of Lyapunov Krasovwkii functionals with polynomial dependence on the the system state (Coutinho \& de Souza 2008).

The paper is organized as follows: In Section 2, we briefly introduce the respiratory system models mentioned above (Vielle \& Chavet 1998), (Kollár \& Turi 2004), (Batzel \& Tran 2000b), (Batzel \& Tran 2000a). In Section 3, we give the main theoretical results on the asymptotic stability of the trivial solution, an estimate of the region of attraction, and exponential estimates for a class of nonlinear system. Finally, in Section 4, we perform the detailed analysis of the stability properties of the corresponding respiratory system models. Section 5 is devoted to the dirrect computation of estimates based on polar coordinates. The paper ends with some concluding remarks.

\section{Mathematical model of the respiratory system}

The human respiratory system can be viewed as an interconnection between a plant which describes the distribution of $\mathrm{O}_{2}$ to the cells and the elimination of the $\mathrm{CO}_{2}$ in the tissues 
of the body, and a physiological controller which regulates the $\mathrm{CO}_{2}$ and/or $\mathrm{O}_{2}$ partial pressure $P$ in the body by acting on the air flow in Lungs with a time lag $h$. Next, we remind four models available in the literature were different structures of the plant and controller are proposed.

\subsection{Mathematical Model I}

In the model introduced in Vielle and Chauvet (Vielle \& Chavet 1998) the plant describes the $\mathrm{CO}_{2}$ exchanges in lungs and tissues and the controller regulates the $\mathrm{CO}_{2}$ partial pressure in lungs with a time lag $h$ :

$$
\begin{aligned}
& \dot{P}_{T}(t)=\kappa_{1} P_{L}(t)-\kappa_{1} P_{T}(t)+\kappa_{2}, \\
& \dot{P}_{L}(t)=\kappa_{3} P_{T}(t)-\kappa_{3} P_{L}(t)-\kappa_{4}\left(P_{L}(t)-\kappa_{5}\right) F\left(P_{L}(t-h)\right),
\end{aligned}
$$

where $\kappa_{1}, \kappa_{2} \kappa_{3}, \kappa_{4}$ and $\kappa_{5}$ are strictly positive constants, $P_{L}$ and $P_{T}$ denote the $C O_{2}$ partial pressures in lungs and tissues. Here, $F(\cdot)$ is the controller function. The following assumptions hold:

(V1) The $\mathrm{CO}_{2}$ partial pressure in lungs is greater than the atmospheric pressure $\kappa_{5}: P_{L}>$ $\kappa_{5}$.

(V2) The controller function $F(\nu)$ is a continuous positive function defined on $\mathbb{R}^{+}$which has zero value for $\nu \leq \nu_{0}$ and a strictly positive derivative for $\nu>\nu_{0}$.

As shown in (Vielle \& Chavet 1998), the equilibrium point $\left(\bar{P}_{T}, \bar{P}_{L}\right)$ of system (1) satisfies:

$$
\begin{aligned}
\bar{P}_{T} & =\bar{P}_{L}+\frac{\kappa_{2}}{\kappa_{1}}, \\
F\left(\bar{P}_{L}\right) & =\frac{\kappa_{2} \kappa_{3}}{\kappa_{1} \kappa_{4}\left[\bar{P}_{L}-\kappa_{5}\right]} .
\end{aligned}
$$

By introducing the new variables $y_{1}(t)=P_{T}(t)-\bar{P}_{T}, y_{2}(t)=P_{L}(t)-\bar{P}_{L}$ and considering that $F\left(P_{L}(t-h)\right) \approx F\left(\bar{P}_{L}\right)+F^{\prime}\left(\bar{P}_{L}\right) y_{2}(t-h)$, system (1) can be rewritten as:

$$
\left(\begin{array}{l}
\dot{y}_{1}(t) \\
\dot{y}_{2}(t)
\end{array}\right)=A_{0}\left(\begin{array}{l}
y_{1}(t) \\
y_{2}(t)
\end{array}\right)+A_{1}\left(\begin{array}{l}
y_{1}(t-h) \\
y_{2}(t-h)
\end{array}\right)+f\left(y_{1}(t), y_{2}(t), y_{1}(t-h), y_{2}(t-h)\right)
$$

where

$$
A_{0}=\left(\begin{array}{cc}
-\kappa_{1} & \kappa_{1} \\
\kappa_{3} & -\left[\kappa_{3}+\kappa_{4} \bar{F}\right]
\end{array}\right), A_{1}=\left(\begin{array}{cc}
0 & 0 \\
0-\kappa_{4} \bar{F}_{p}\left[\bar{P}_{L}-\kappa_{5}\right]
\end{array}\right)
$$


and

$$
f\left(y_{1}(t), y_{2}(t), y_{1}(t-h), y_{2}(t-h)\right)=\left(\begin{array}{c}
0 \\
-\kappa_{4} \bar{F}_{p} y_{2}(t) y_{2}(t-h)
\end{array}\right),
$$

with $\bar{F}=F\left(\bar{P}_{L}\right), \bar{F}_{p}=F^{\prime}\left(\bar{P}_{L}\right)$.

The following conditions for the stability of the linear part of system (4) known as the nominal system is asymptotically stable are available:

Lemma 1 (Vielle \& Chavet 1998) If $\kappa_{1}, \kappa_{3}, \kappa_{4} \bar{F}, \kappa_{4} \bar{F}_{p}\left[\bar{P}_{L}-\kappa_{5}\right]$ are strictly positive coefficients, and $\bar{F}<\bar{F}_{p}\left[\bar{P}_{L}-\kappa_{5}\right]$, then there exists $h_{0}>0$ such that the nominal system of (41) is asymptotically stable for $h \in\left[0, h_{o}\right)$ and unstable for $h \geq h_{0}$.

Let us consider the following Hill controller function

$$
F(\nu)=V_{m} \frac{\nu^{n}}{\theta^{n}+\nu^{n}}, \quad \nu \geq 0,
$$

where $V_{m}>0$ is the maximum air flow, $n>0$ is the Hill coefficient, and $\theta>0$ is the Hill parameter. Observe that function (7) satisfies (V2) if $\nu \geq \nu_{0}=0$.

Consider the parameters of system (44) and of the controller function (17) given in (Vielle \& Chavet 1998): $\kappa_{1}=0.0067, \kappa_{3}=0.1448, \kappa_{4}=3200^{-1}, \kappa_{5}=0.3, \theta=48.6, \quad V m=1330$, and $n=13.7$.

Using (21) and (3) we obtain that the equilibrium is $\left(\bar{P}_{T}, \bar{P}_{L}\right)=(47.27,39.97)$. Substituting into (77) implies that $F\left(\bar{P}_{L}\right)=85.38 \mathrm{mls}^{-1}$ and $F^{\prime}\left(\bar{P}_{L}\right)=27.39$.

By Lemma 1 we have that $\omega_{o}=0.2897$ and $h_{o}=7.249$, hence the nominal system of (4) is asymptotically stable for all $h \in[0,7.249)$ and unstable for all $h \geq 7.249$.

\subsection{Mathematical Model II}

In Kollar and Turi (Kollár \& Turi 2004) the plant describes $\mathrm{CO}_{2}$ and $\mathrm{O}_{2}$ arterial partial pressures in lungs while the controller regulates the $\mathrm{CO}_{2}$ partial pressure and the $\mathrm{O}_{2}$ partial pressure in lungs with a time lag $h$ :

$$
\begin{aligned}
& \dot{P}_{c}(t)=1-\alpha P_{c}(t) F\left(P_{c}(t-h), P_{o}(t-h)\right), \\
& \dot{P}_{o}(t)=1-\beta P_{o}(t) F\left(P_{c}(t-h), P_{o}(t-h)\right),
\end{aligned}
$$

where $P_{c}$ is the $\mathrm{CO}_{2}$ partial pressures in lungs, $P_{o}$ is the $\mathrm{O}_{2}$ partial pressures in lungs, $\alpha$ and $\beta$ are positive constants and $F(\cdot, \cdot)$ is the controller function with a transport delay. It is biologically realistic to assume that the controller function satisfies the following properties: 
(K1) $F\left(\nu_{1}, \nu_{2}\right) \geq 0$ and $F(0,0)=0$,

(K2) $F\left(\nu_{1}, \nu_{2}\right)$ is differentiable, and

(K3) $F_{\nu_{1}}=\partial F\left(\nu_{1}, \nu_{2}\right) / \partial \nu_{1}>0, F_{\nu_{2}}=\partial F\left(\nu_{1}, \nu_{2}\right) / \partial \nu_{2}>0$.

The unique positive equilibrium $\left(\bar{P}_{c}, \bar{P}_{o}\right)$ of system (8) is such that

$$
\begin{aligned}
& 0=1-\alpha F\left(\bar{P}_{c}, \bar{P}_{o}\right) \bar{P}_{c}, \\
& 0=1-\beta F\left(\bar{P}_{c}, \bar{P}_{o}\right) \bar{P}_{o} .
\end{aligned}
$$

By introducing the new state variables $y_{1}(t)=P_{c}(t)-\bar{P}_{c}, y_{2}(t)=P_{o}(t)-\bar{P}_{o}$, and considering a first order Taylor series approximation of $F\left(P_{c}(t), P_{o}(t)\right)$, the system (8) can be rewritten as

$$
\left(\begin{array}{l}
\dot{y}_{1}(t) \\
\dot{y}_{2}(t)
\end{array}\right)=A_{0}\left(\begin{array}{l}
y_{1}(t) \\
y_{2}(t)
\end{array}\right)+A_{1}\left(\begin{array}{l}
y_{1}(t-h) \\
y_{2}(t-h)
\end{array}\right)+f\left(y_{1}(t), y_{2}(t), y_{1}(t-h), y_{2}(t-h)\right)
$$

where

$$
A_{0}=\left(\begin{array}{cc}
-\alpha \bar{F} & 0 \\
0 & -\beta \bar{F}
\end{array}\right), A_{1}=\left(\begin{array}{c}
-\alpha \bar{P}_{c} \bar{F}_{P_{c}}-\alpha \bar{P}_{c} \bar{F}_{P_{o}} \\
-\beta \bar{P}_{o} \bar{F}_{P_{c}}-\beta \bar{P}_{o} \bar{F}_{P_{o}}
\end{array}\right),
$$

and

$$
f\left(y_{1}(t), y_{2}(t), y_{1}(t-h), y_{2}(t-h)\right)=\left(\begin{array}{l}
-\alpha \bar{F}_{P_{c}} y_{1}(t) y_{1}(t-h)-\alpha \bar{F}_{P_{o}} y_{1}(t) y_{2}(t-h) \\
-\beta \bar{F}_{P_{c}} y_{2}(t) y_{1}(t-h)-\beta \bar{F}_{P_{o}} y_{2}(t) y_{2}(t-h)
\end{array}\right),
$$

with $\bar{F}=F\left(\bar{P}_{c}, \bar{P}_{o}\right), \bar{F}_{P_{c}}=F_{P_{c}}\left(\bar{P}_{c}, \bar{P}_{o}\right)$, and $\bar{F}_{P_{o}}=F_{P_{o}}\left(\bar{P}_{c}, \bar{P}_{o}\right)$.

The following stability result for the nominal system is given:

Lemma 2 (Cooke 83 Turi 1994) If $\bar{F}<\bar{P}_{c} \bar{F}_{P_{c}}+\bar{P}_{o} \bar{F}_{P_{o}}$, then there exists $h_{0}>0$ such that the nominal system of (10) is asymptotically stable for $h \in\left[0, h_{0}\right)$ and unstable for $h \geq h_{0}$.

In the following we analyze system (10) using the parameters $\alpha=0.5, \beta=0.8$ and the controller function

$$
F\left(\nu_{1}, \nu_{2}\right)=G_{P} e^{-0.05\left(100-\nu_{2}\right)}\left(\nu_{1}-I_{P}\right)
$$

proposed in (Kollár \& Turi 2004). Here $G_{p}=0.14$ and $I_{P}=0$ are the control gains and cutoff thresholds, respectively.

It follows from (9) that the the equilibrium is $\left(\bar{P}_{c}, \bar{P}_{o}\right)=(29.1842,18.2401)$. Consequently, we obtain from (13) that $\bar{F}_{P_{c}}=0.0023, \bar{F}_{P_{o}}=0.0034$, and $\bar{F}=0.0685$.

It follows from Lemma 2 that $h_{o}=30.8$, hence the nominal system (10) is asymptotically stable for all $h \in[0,30.8)$ and unstable for all $h \geq 30.8$. 


\subsection{Mathematical Model III}

In Batzel and Tran (Batzel \& Tran 2000b) the plant describes $\mathrm{CO}_{2}$ and $\mathrm{O}_{2}$ arterial partial pressures in lungs and the controller regulates the $\mathrm{CO}_{2}$ partial pressure and the $\mathrm{O}_{2}$ partial pressure in lungs with a time lag $h$ :

$$
\begin{aligned}
& \dot{P}_{c}(t)=a_{1}-a_{2} P_{c}(t)-a_{3} P_{c}(t) F\left(P_{c}(t-h), P_{o}(t-h)\right), \\
& \dot{P}_{o}(t)=b_{1}-b_{2} P_{o}(t)-b_{3} P_{o}(t) F\left(P_{c}(t-h), P_{o}(t-h)\right) .
\end{aligned}
$$

The state variables and the assumptions are the name as those in the previous section. The system (14) has a unique positive equilibrium $\left(\bar{P}_{c}, \bar{P}_{o}\right)$ such that

$$
\begin{aligned}
& 0=a_{1}-a_{2} \bar{P}_{c}-a_{3} F\left(\bar{P}_{c}, \bar{P}_{o}\right) \bar{P}_{c} \\
& 0=b_{1}-b_{2} \bar{P}_{o}-b_{3} F\left(\bar{P}_{c}, \bar{P}_{o}\right) \bar{P}_{o} .
\end{aligned}
$$

By introducing the new state variables $y_{1}(t)=P_{c}(t)-\bar{P}_{c}, y_{2}(t)=P_{o}(t)-\bar{P}_{o}$ and considering a first order Taylor series approximation of $F\left(P_{c}(t), P_{o}(t)\right)$, the system (14) can be rewritten as

$$
\left(\begin{array}{l}
\dot{y}_{1}(t) \\
\dot{y}_{2}(t)
\end{array}\right)=A_{0}\left(\begin{array}{l}
y_{1}(t) \\
y_{2}(t)
\end{array}\right)+A_{1}\left(\begin{array}{l}
y_{1}(t-h) \\
y_{2}(t-h)
\end{array}\right)+f\left(y_{1}(t), y_{2}(t), y_{1}(t-h), y_{2}(t-h)\right)
$$

where

$$
A_{0}=\left(\begin{array}{cc}
-a_{2}-a_{3} \bar{F} & 0 \\
0 & -b_{2}-b_{3} \bar{F}
\end{array}\right), A_{1}=\left(\begin{array}{c}
-a_{3} \bar{P}_{c} \bar{F}_{P_{c}}-a_{3} \bar{P}_{c} \bar{F}_{P_{o}} \\
-b_{3} \bar{P}_{o} \bar{F}_{P_{c}}-b_{3} \bar{P}_{o} \bar{F}_{P_{o}}
\end{array}\right)
$$

and

$$
f\left(y_{1}(t), y_{2}(t), y_{1}(t-h), y_{2}(t-h)\right)=\left(\begin{array}{l}
-a_{3} \bar{F}_{P_{c}} y_{1}(t) y_{1}(t-h)-a_{3} \bar{F}_{P_{o}} y_{1}(t) y_{2}(t-h) \\
-b_{3} \bar{F}_{P_{c}} y_{2}(t) y_{1}(t-h)-b_{3} \bar{F}_{P_{o}} y_{2}(t) y_{2}(t-h)
\end{array}\right),
$$

with $\bar{F}=F\left(\bar{P}_{c}, \bar{P}_{o}\right), \bar{F}_{P_{c}}=F_{P_{c}}\left(\bar{P}_{c}, \bar{P}_{o}\right)$, and $\bar{F}_{P_{o}}=F_{P_{o}}\left(\bar{P}_{c}, \bar{P}_{o}\right)$.

The stability of the nominal system has the following characterization:

Lemma 3 (Batzel \& Tran 2000b) If $K_{1} K_{3}-\left(K_{1} K_{4}+K_{2} K_{3}\right)<0$, then there exists $h_{0}>0$ such that the nominal system of (16) is asymptotically stable for $h \in\left[0, h_{0}\right)$ and unstable for $h \geq h_{0}$. Here, $K_{1}=a_{2}+a_{3} \bar{F}, K_{2}=a_{3} \bar{P}_{c} \bar{F}_{P_{c}}, K_{3}=b_{2}+b_{3} \bar{F}$ and $K_{4}=b_{3} \bar{P}_{o} \bar{F}_{P_{o}}$.

In the following, we examine system (16) by setting $a_{2}=9.223, a_{3}=0.2187, b_{2}=0.5178$, $b_{3}=0.28$, and the controller function

$$
F\left(\nu_{1}, \nu_{2}\right)=F_{p}\left(\nu_{1}, \nu_{2}\right)+F_{c}\left(\nu_{1}, \nu_{2}\right)
$$


proposed in (Batzel \& Tran 2000b). Here

$$
\begin{aligned}
& F_{p}\left(\nu_{1}, \nu_{2}\right)=G_{p} e^{-0.05\left(146-\nu_{2}\right)}\left(\nu_{1}-I_{p}\right), \\
& F_{c}\left(\nu_{1}, \nu_{2}\right)=K_{V c 1}+K_{V c 2}\left(\nu_{1}-I_{c}\right),
\end{aligned}
$$

where $G_{p}=45$ is the control gain, $I_{p}=35$ is the cutoff thresholds and $K_{V c 1}=3, K_{V c 2}=0.5$ are positive constants.

It follows from (15) that the equilibrium is $\left(\bar{P}_{c}, \bar{P}_{o}\right)=(39.57,48.46)$. One obtains from (19) that $\bar{F}_{P_{c}}=0.8429, \bar{F}_{P_{o}}=0.0783, \bar{F}=6.852$.

By Lemma 3 we have that $\omega_{0}=1.765$ and $h_{0}=95$, hence the nominal of system (16) is asymptotically stable for all $h \in[0,95)$ and unstable for all $h \geq 95$.

\subsection{Mathematical Model IV}

In (Batzel \& Tran 2000b) a third state equation describing the $\mathrm{CO}_{2}$ partial pressure in brain denoted by $\sigma(t)$ is introduced in the model of the previous section:

$$
\begin{aligned}
& \dot{P}_{c}(t)=a_{1}-a_{2} P_{c}(t)-a_{3} P_{c}(t) F\left(P_{c}(t-h), P_{o}(t-h), \sigma(t)\right), \\
& \dot{P}_{o}(t)=b_{1}-b_{2} P_{o}(t)-b_{3} P_{o}(t) F\left(P_{c}(t-h), P_{o}(t-h), \sigma(t)\right), \\
& \dot{\sigma}(t)=c_{1}+c_{2} P_{c}(t-h)-c_{2} \sigma(t),
\end{aligned}
$$

The assumptions on the controller function are now:

(T1) $F\left(\nu_{1}, \nu_{2}, \nu_{3}\right) \geq 0$ and $F(0,0,0)=0$,

(T2) $F\left(\nu_{1}, \nu_{2}, \nu_{3}\right)$ is differentiable, and

(T3) $F_{\nu_{1}}=\frac{\partial F\left(\nu_{1}, \nu_{2}, \nu_{3}\right)}{\partial \nu_{1}}>0, F_{\nu_{2}}=\frac{\partial F\left(\nu_{1}, \nu_{2}, \nu_{3}\right)}{\partial \nu_{2}}>0 F_{\nu_{3}}=\frac{\partial F\left(\nu_{1}, \nu_{2}, \nu_{3}\right)}{\partial \nu_{3}}>0$.

As shown in (Batzel \& Tran 2000a), system (21) has a unique positive equilibrium $\left(\bar{P}_{c}, \bar{P}_{o}, \bar{\sigma}\right)$ such that

$$
\begin{aligned}
& 0=a_{1}-a_{2} \bar{P}_{c}-a_{3} F\left(\bar{P}_{c}, \bar{P}_{o}\right) \bar{P}_{c}, \\
& 0=b_{1}-b_{2} \bar{P}_{o}-b_{3} F\left(\bar{P}_{c}, \bar{P}_{o}\right) \bar{P}_{o} \\
& 0=c_{1}+c_{2} \bar{P}_{c}-c_{2} \bar{\sigma} .
\end{aligned}
$$

By introducing the new state variables $y_{1}(t)=P_{c}(t)-\bar{P}_{c}, y_{2}(t)=P_{o}(t)-\bar{P}_{o}, y_{3}(t)=\sigma(t)-\bar{\sigma}$ and considering a fist order Taylor series approximation of $F\left(\nu_{1}, \nu_{2}, \nu_{3}\right)$, the system (21) can be rewritten as

$$
\left(\begin{array}{l}
\dot{y}_{1}(t) \\
\dot{y}_{2}(t)
\end{array}\right)=A_{0}\left(\begin{array}{l}
y_{1}(t) \\
y_{2}(t)
\end{array}\right)+A_{1}\left(\begin{array}{l}
y_{1}(t-h) \\
y_{2}(t-h)
\end{array}\right)+f\left(y_{1}(t), y_{2}(t), y_{1}(t-h), y_{2}(t-h)\right)
$$


where



and

$$
\begin{aligned}
& f\left(y_{1}(t), y_{2}(t), y_{3}(t), y_{1}(t-h), y_{2}(t-h), y_{3}(t-h)\right)= \\
& \qquad\left(\begin{array}{c}
-a_{3} \bar{F}_{P_{c}} y_{1}(t) y_{1}(t-h)-a_{3} \bar{F}_{P_{o}} y_{1}(t) y_{2}(t-h)-a_{3} \bar{F}_{\sigma} y_{1}(t) y_{3}(t) \\
-b_{3} \bar{F}_{P_{c}} y_{2}(t) y_{1}(t-h)-b_{3} \bar{F}_{P_{o}} y_{2}(t) y_{2}(t-h)-b_{3} \bar{F}_{\sigma} y_{2}(t) y_{3}(t) \\
0
\end{array}\right),
\end{aligned}
$$

with $\bar{F}=F\left(\bar{P}_{c}, \bar{P}_{o}, \bar{\sigma}\right), \bar{F}_{P_{c}}=F_{P_{c}}\left(\bar{P}_{c}, \bar{P}_{o}, \bar{\sigma}\right), \bar{F}_{P_{o}}=F_{P_{o}}\left(\bar{P}_{c}, \bar{P}_{o}, \bar{\sigma}\right)$, and $\bar{F}_{\sigma}=F_{\sigma}\left(\bar{P}_{c}, \bar{P}_{o}, \bar{\sigma}\right)$. Using analytical methods to obtain stability conditions for the nominal systems of the previous models is complicated. However, we can determine the stability with the help of a Mikhailov hodograph (Kolmanosvkii \& Myshkis 1999).

In the following we examine system (23) by setting $a_{2}=9.2233, a_{3}=0.2187, b_{2}=0.5178$, $b_{3}=0.28, c_{1}=8.1871, c_{2}=0.8333$ and the controller function

$$
F\left(\nu_{1}, \nu_{2}, \nu_{3}\right)=F_{P}\left(\nu_{1}, \nu_{2}, \nu_{3}\right)+F_{C}\left(\nu_{1}, \nu_{2}, \nu_{3}\right)
$$

given in (Batzel \& Tran 2000a). Here $F_{P}\left(\nu_{1}, \nu_{2}, \nu_{3}\right)=G_{P} e^{-0.05\left(146-\nu_{2}\right)}\left(\nu_{1}-I_{p}\right), F_{C}\left(\nu_{1}, \nu_{2}, \nu_{3}\right)=$ $G_{C}\left(\nu_{3}-\frac{c_{1}}{c_{2}}-I_{C}\right)$,

where $G_{P}=45$ and $G_{C}=1.2$ are control gains and $I_{P}=35 I_{C}=35$ are cutoff thresholds. It follows from (22) that the equilibrium is $\left(\bar{P}_{c}, \bar{P}_{o}, \bar{\sigma}\right)=(39.41,48.74,49.23)$. We obtain from (26) that $\bar{F}_{P_{c}}=0.3477, \bar{F}_{P_{o}}=0.0767, \bar{F}_{\sigma}=1.2, \bar{F}=6.82, \bar{F}_{P}=1.5335, \bar{F}_{C}=5.2865$ and the approximate critical delay is $h_{0}=95.71$.

\section{Tools for the stability analysis of the trivial solution}

In what follows we introduce results concerning the stability properties of the solutions of nonlinear systems achieved in the framework of Lyapunov-Krasovskii functionals of complete type.

We analyze nonlinear systems of the form

$$
\begin{aligned}
& \dot{y}(t)=A_{0} y(t)+A_{1} y(t-h)+f(y(t), y(t-h)), \\
& y(\theta)=\psi(\theta), \quad \theta \in[-h, 0]
\end{aligned}
$$


where $A_{0}, A_{1} \in \mathbb{R}^{n \times n}$ are given matrices, $h$ is the delay and $\psi \in \mathfrak{C}$ is the initial function.

We denote by $y(t, \psi)$ the solution of the system with initial condition $\psi$, by $y_{t}(\psi)=\{y(t+$ $\tau, \psi): \tau \in[-h, 0]\}$ the segment of trajectory of the system and by $\mathfrak{C}:=C\left([-h, 0], \mathbb{R}^{n}\right)$ the Banach space with norm $\|\psi\|_{h}:=\max _{\tau \in[-h, 0]}\|\psi(\tau)\|$.

We consider the following assumptions for system (27):

(A1) the nominal system

$$
\dot{x}(t)=A_{0} x(t)+A_{1} x(t-h),
$$

is exponentially stable.

The function $f\left(z_{0}, z_{1}\right)$ satisfies

(A2) $f(\overline{0}, \overline{0})=\overline{0}, \overline{0} \in \mathbb{R}^{n}$,

(A3) $f\left(z_{0}, z_{1}\right), z_{0}, z_{1} \in \mathbb{R}^{n}$, satisfies a Lipschitz condition in a neighborhood of the origin, (A4) for any $\gamma>0$ there exists $\varepsilon=\varepsilon(\gamma)>0$ such that if $\left\|\left(z_{0}, z_{1}\right)\right\|<\varepsilon$, then $\left\|f\left(z_{0}, z_{1}\right)\right\|<$ $\gamma\left\|\left(z_{0}, z_{1}\right)\right\|_{Q}$. Here

$$
\left\|\left(z_{0}, z_{1}\right)\right\|_{Q}^{2}=\left(\begin{array}{c}
z_{0} \\
z_{1}
\end{array}\right)^{T} \underbrace{\left(\begin{array}{c}
Q_{11} Q_{12} \\
Q_{12} Q_{22}
\end{array}\right)}_{Q}\left(\begin{array}{c}
z_{0} \\
z_{1}
\end{array}\right),
$$

where $Q \in \mathbb{R}^{2 n \times 2 n}$ is a positive definite matrix. Observe that if $Q=I_{n}$, then $\left\|\left(z_{0}, z_{1}\right)\right\|_{Q}=$ $\left\|\left(z_{0}, z_{1}\right)\right\| . I_{n}$ is the identity matrix in $\mathbb{R}^{n \times n}$.

\subsection{Stability of the trivial solution of nonlinear systems}

We now obtain asymptotic stability conditions for the trivial solution of the nonlinear system (27) based on the fact that a Lyapunov-Krasovskii functional of complete type $v\left(y_{t}\right)$ admits a quadratic bound and that its time derivative $\dot{v}\left(y_{t}\right)$ along the trajectories of system (27) is negative (Kharitonov \& Zhabko 2003).

Lemma 4 Consider a nonlinear system of the form (27). Let positive definite matrices $W_{i} \in \mathbb{R}^{n \times n}, i=0,1,2$ and a symmetric real matrix $Z \in \mathbb{R}^{n \times n}$ such that

$$
\left(\begin{array}{cc}
W_{0} & Z A_{1} \\
A_{1}^{T} Z & W_{1}
\end{array}\right)>0
$$

be given. Then the trivial solution of the nonlinear system (27) is asymptotically stable if 
there exists $\gamma>0$ such that

$$
\gamma\left(\begin{array}{ccc}
\frac{\Gamma}{h} Q_{11}+\frac{u_{o z}}{h} I_{n} & \frac{\Gamma}{h} Q_{12} & 0 \\
\frac{\Gamma}{h} Q_{12} & \frac{\Gamma}{h} Q_{22} & 0 \\
0 & 0 & a_{1} u_{1} I_{n}
\end{array}\right)<\left(\begin{array}{ccc}
\frac{W_{0}}{h} & \frac{Z A_{1}}{h} & 0 \\
\frac{A_{1}^{T} Z}{h} & \frac{W_{1}}{h} & 0 \\
0 & 0 & W_{2}
\end{array}\right),
$$

where $\Gamma=u_{o z} / h+a_{1} u_{1}, a_{1}=\left\|A_{1}\right\|, u_{o z}=\|U(0, W)-Z\|, u_{1}=\max _{\tau \in[0, h]}\{\|U(\tau, W)\|\}$, $Q_{11}, Q_{22}, Q_{12}, I_{n} \in \mathbb{R}^{n \times n}$. Here, $U(\tau, W)$ is the Lyapunov matrix defined in Remark 10 of the appendix.

Proof. It follows from Assumption A1 that the nominal system (28) is exponentially stable. Then given a quadratic functional of the form

$$
w\left(x_{t}\right)=x^{T}(t) W_{0} x(t)+x^{T}(t-h) W_{1} x(t-h)+2 x^{T}(t) Z A_{1} x(t-h)+\int_{-h}^{0} x^{T}(t+\tau) W_{2} x(t+\tau) d \tau,
$$

where $W_{i}, i=0,1,2$, are positive definite matrices, there exists a unique LyapunovKrasovskii functional of complete type $v\left(x_{t}\right)$ described by (52) in the appendix such that the time derivative of the functional along the trajectories of the nominal system (28) is $\dot{v}\left(x_{t}\right)=-w\left(x_{t}\right)$ (Mondié et al. 2005).

Now, we observe that the time derivative of the functional along the trajectories of system (27) is

$$
\dot{v}\left(y_{t}\right)=-w\left(y_{t}\right)+2 f^{T}(y(t), y(t-h))\left[[U(0, W)-Z] y(t)+\int_{-h}^{0} U(-h-\theta, W) A_{1} y(t+\tau) d \tau\right] .
$$

It follows from Assumption A4 that for every $\gamma>0$ the function $f\left(z_{0}, z_{1}\right)$ satisfies $\left\|f\left(z_{0}, z_{1}\right)\right\|<$ $\gamma\left\|\left(z_{0}, z_{1}\right)\right\|_{Q}$. Substituting this inequality into (30) leads to

$$
\begin{aligned}
\dot{v}\left(y_{t}\right) & \leq-w\left(y_{t}\right)+2 \gamma u_{o z}\|y(t)\|\|(y(t), y(t-h))\|_{Q}+2 \gamma a_{1} u_{1} \int_{-h}^{0}\|y(t+\tau)\|\|(y(t), y(t-h))\|_{Q} d \tau \\
& \leq-\int_{-h}^{0}\left(\begin{array}{c}
y(t) \\
y(t-h) \\
y(t+\theta)
\end{array}\right) M\left(\begin{array}{c}
y(t) \\
y(t-h) \\
y(t+\theta)
\end{array}\right) d \theta, \quad t \geq 0,
\end{aligned}
$$

where

$$
M=\left(\begin{array}{ccc}
\frac{W_{0}}{h} \frac{Z A_{1}}{h} & 0 \\
\frac{A_{1}^{T} Z}{h} & \frac{W_{1}}{h} & 0 \\
0 & 0 & W_{2}
\end{array}\right)-\gamma\left(\begin{array}{ccc}
\frac{\Gamma}{h} Q_{11}+\frac{u_{z o}}{h} I_{n} & \frac{\Gamma}{h} Q_{12} & 0 \\
\frac{\Gamma}{h} Q_{12} & \frac{\Gamma}{h} Q_{22} & 0 \\
0 & 0 & a_{1} u_{1} I_{n}
\end{array}\right),
$$

with $\Gamma=a_{1} u_{1} h+u_{o z}, a_{1}=\left\|A_{1}\right\|, u_{o z}=\|U(0, W)-Z\|, u_{1}=\max _{\tau \in[0, h]}\{\|U(\tau, W)\|\}$. 
Now, if there exists $\gamma>0$ such that $M>0$, we have that

$$
\dot{v}\left(y_{t}\right) \leq-\int_{-h}^{0} \lambda_{\min }(M)\|(y(t), y(t-h), y(t+\tau))\|^{2} d \tau \leq-\zeta\|y(t)\|^{2}, \quad t \geq 0
$$

where $\zeta=\lambda_{\min }(M) / h$.

In addition, the functional $v\left(y_{t}\right)$ satisfies

$$
\alpha_{1}\|y(t)\|^{2} \leq v\left(y_{t}\right) \leq \alpha_{2}\left\|y_{t}\right\|_{h}^{2}, \quad \forall t \geq 0
$$

with $\alpha_{1}$ and $\alpha_{2}$ given by (56) and (57) of Theorem 111 of the Appendix, respectively.

Thus, it follows from (32) and (33) that the functional $v\left(y_{t}\right)$ satisfies the conditions of the Krasovskii asymptotic stability theorem (Krasovskii 1956) and we conclude that the trivial solution of system (27) is asymptotically stable.

\subsection{Estimate of the region of attraction}

The asymptotic stability of the equilibrium of the system is indeed a crucial property. But it is important from a practical point of view to know the set of initial conditions of system (27) that generates trajectories that converge to the equilibrium as $t$ approaches infinity. This set is called the region of attraction of the equilibrium.

Definition 5 Let the trivial solution of system (27) be asymptotically stable. The set

$$
R_{A}=\{\psi \in \mathfrak{C}: y(t, \psi) \text { is defined } \forall t \geq 0 \text { and } y(t, \psi) \underset{t \rightarrow \infty}{\longrightarrow} 0\}
$$

is the region of attraction of the trivial solution of system (27).

Definition 6 Let the trivial solution of system (27) be asymptotically stable. A set $\Omega \subset \mathfrak{C}$ is said to be an estimate of the region of attraction of the trivial solution of system (27) if

i. $0_{h} \in \Omega$,

ii. $\Omega \subset R_{A}$.

The result stated below provides an estimate of the region of attraction of the trivial solution of system (27): 
Theorem 7 Consider a system of the form (27) and let $\gamma$ be a positive constant such that (29) holds. Then, the set

$$
\Omega=\left\{\psi \in \mathfrak{C}:\|\psi\|_{h}<\nu\right\}
$$

with $\nu=\sqrt{\frac{\alpha_{1}}{\alpha_{2}}} \varepsilon / 2$ is an estimate of the region of attraction of the trivial solution of the system. Here, the constant $\varepsilon=\varepsilon(\gamma)$ is obtained from Assumption $\mathbf{A} 4$ and the constants $\alpha_{1}$ and $\alpha_{2}$ are given in (56) and (57) of Theorem 11 of the appendix.

Proof. We prove that the set (34) satisfies the Conditions of Definition 6 , First, we observe that the set (34) contains the trivial solution, i.e., $\psi=0_{h} \in \Omega$.

Now, we shows that for any initial condition in the set (34) the solution of system (27) converge to zero as $t$ approaches infinity.

It follows from Lemma 4 and Assumption A4 that for $\gamma>0$ such that (29) holds, there exists $\varepsilon=\varepsilon(\gamma)>0$ such that

$$
\|(y(t), y(t-h))\|<\varepsilon \Rightarrow \dot{v}\left(y_{t}\right)<0, \quad \forall t \geq 0 .
$$

This implies that the functional $v\left(y_{t}\right)$ is decreasing for all $t \geq 0$, hence

$$
v\left(y_{t}\right) \leq v(\psi), \quad \forall t \geq 0
$$

It follows from equation (54) of Theorem 11 of the Appendix that

$$
\alpha_{1}\|y(t)\|^{2} \leq v\left(y_{t}\right) \leq v(\psi) \leq \alpha_{2}\|\psi\|_{h}^{2}, \quad \forall t \geq 0
$$

therefore, for any $\psi \in \Omega$ we have that

$$
\|y(t)\| \leq \frac{\varepsilon}{2}, \quad \forall t \geq 0
$$

Moreover,

$$
\|y(t)\| \leq \frac{\varepsilon}{2}, \quad \Rightarrow \quad \dot{v}\left(y_{t}\right)<0, \quad \forall t \geq 0 .
$$

Thus,

$$
\lim _{t \rightarrow \infty} y(t, \psi)=0, \quad \forall \psi \in \Omega .
$$

We conclude that the set described by (34) is an estimate of the region of attraction of the trivial solution of system (27). 


\subsection{Exponential estimate of the system response}

In this section, we obtain an exponential estimate for the solution of system (27) whose initial condition $\psi$ belongs to the estimate of the region of attraction.

Corollary 8 Consider a system of the form (27). Then, for any $\psi \in \Omega$ the solution $y(t, \psi)$ of system (27) satisfies the following exponential estimate

$$
\|y(t, \psi)\| \leq \delta\|\psi\|_{h} e^{-\varsigma t}, \quad t \geq 0
$$

where $\delta=\sqrt{\frac{\alpha_{2}}{\alpha_{1}}}$ and $2 \varsigma=\min \left\{\lambda_{\min }\left(R_{1}\right) / \eta_{1}, \lambda_{\min }\left(R_{2}\right) / \eta_{2}\right\}, \eta_{1}=u_{z o}+h u_{1} a_{1}, \eta_{2}=u_{1} a_{1}+$ $w_{1}+h w_{2}+h u_{1} a_{1}^{2}, R_{2}=W_{2}-\gamma a_{1} u_{1} I_{n}$ and $R_{1}=\left(\begin{array}{cc}W_{0}-\gamma\left[\Gamma Q_{11}+u_{o z} I_{n}\right] Z A_{1}-\gamma \Gamma Q_{12} \\ A_{1}^{T} Z-\gamma \Gamma Q_{12} & W_{1}-\gamma \Gamma Q_{22}\end{array}\right)$.

Proof. We know that

$$
\begin{aligned}
\dot{v}\left(y_{t}\right) \leq & -\left(y^{T}(t) y^{T}(t-h)\right) R_{1}\left(\begin{array}{l}
y(t) \\
y(t-h)
\end{array}\right) \\
& -\int_{-h}^{0} y^{T}(t+\tau) R_{2} y(t+\tau) d \tau \\
\leq & -\lambda_{\min }\left(R_{1}\right)\|y(t)\|^{2}-\lambda_{\min }\left(R_{2}\right) \int_{-h}^{0}\|y(t+\tau)\|^{2} d \tau, \quad t \geq 0 .
\end{aligned}
$$

It follows from (52) that

$$
v\left(y_{t}\right) \leq \eta_{1}\|y(t)\|^{2}+\eta_{2} \int_{-h}^{0}\|y(t+\tau)\|^{2} d \tau, \quad t \geq 0 .
$$

Now, using (37) and (38) we have that

$$
\frac{d v\left(y_{t}\right)}{d t}+2 \varsigma v\left(y_{t}\right) \leq 0, \quad t \geq 0
$$

where $2 \varsigma=\min \left\{\lambda_{\min }\left(R_{1}\right) / \eta_{1}, \lambda_{\min }\left(R_{2}\right) / \eta_{2}\right\}$. Multiplying by $e^{-2 \varsigma t}$ both sides of (39) we get

$$
\frac{d}{d t}\left(e^{2 \varsigma t} v\left(y_{t}\right)\right)<0, \quad t \geq 0
$$

Integrating this inequality from 0 to $t$ and using the fact that the solution goes to zero as $t$ tends to infinity we get

$$
v\left(y_{t}\right) \leq e^{-2 \varsigma t} v(\psi), \quad t \geq 0
$$

thus (56) and (57) imply that

$$
\alpha_{1}\|y(t)\|^{2} \leq v\left(y_{t}\right) \leq e^{-2 \varsigma t} v(\psi) \leq \alpha_{2} e^{-2 \varsigma t}\|\psi\|, \quad t \geq 0 .
$$

Finally, recalling that $y(t)$ depends on $\psi$, i.e. $y(t)=y(t, \psi)$, we conclude that $\|y(t, \psi)\| \leq$ $\sqrt{\frac{\alpha_{2}}{\alpha_{1}}}\|\psi\|_{h} e^{-\varsigma t}, \quad t \geq 0$. 


\section{Analysis of the human respiratory system}

We now analyze the stability properties of the respiratory systems described in Section 2. Due to space limitations we provide the detailed analysis for model I and we give final results for the remaining ones.

The asymptotic stability and exponential stability are equivalent for time delay linear systems because theirs solutions are of exponential type ( $\mathrm{Gu}$, Kharitonov \& Chen 2003). Therefore, the Assumption A1 holds in all cases. It is trivial to prove that the Assumptions A2 and A3 hold in all the models under study.

In the following subsections we proceed as follows for each respiratory model:

First, we prove that Assumption A4 is satisfied,

Second, we propose positive definite matrices $W_{i} \in \mathbb{R}^{n \times n}, i=0,1,2$ and a symmetric real matrix $Z \in \mathbb{R}^{n \times n}$ such that

$$
\left(\begin{array}{cc}
W_{0} & Z A_{1} \\
A_{1}^{T} Z & W_{1}
\end{array}\right)>0
$$

holds. Following the methodology proposed in (Garcia-Lozano \& Kharitonov 2006) we compute the Lyapunov matriz $U(\tau, W)$ associated to $W=W_{0}+W_{1}+h W_{2}-A_{0}^{T} Z-Z A_{0}$. Third, we obtain a positive constant $\gamma$ such that the LMI (29) is feasible. Substituting $\gamma$ into Assumption (A4) yields $\varepsilon=\varepsilon(\gamma)$.

Fourth, we get the quadratic bounds $\alpha_{1}$ and $\alpha_{2}$ using Theorem 11 of the appendix. The estimate of the region of attraction $\Omega$ of the trivial solution of the respiratory system follows from substituting these quadratic bounds and $\gamma$ into (34).

Finally, an exponential estimate of the solution is obtained from Corollary 8 ,

Detailed computations for Model I

First, for the nonlinear part (66) we have that

$$
\left\|f\left(z_{0}, z_{1}, z_{2}, z_{3}\right)\right\|^{2}<\frac{\left(\kappa_{4} \bar{F}_{p}\right)^{2} \varepsilon^{2}}{4}\left\|\left(z_{0}, z_{1}, z_{2}, z_{3}\right)\right\|_{Q}^{2}
$$

where $Q=I_{4}$ is the identity matriz in $\mathbb{R}^{4 \times 4}$. Therefore, for any $\gamma>0$ there exits $\varepsilon=\varepsilon(\gamma)$ solution of the equation

$$
\varepsilon=\frac{2 \gamma}{\kappa_{4} \bar{F}_{p}}
$$

such that $\left\|f\left(z_{0}, z_{1}, z_{2}, z_{3}\right)\right\|<\gamma\left\|\left(z_{0}, z_{1}, z_{2}, z_{3}\right)\right\|_{Q}$ if $\left\|\left(z_{0}, z_{1}, z_{2}, z_{3}\right)\right\|<\varepsilon$. 
Second, choosing $h=0.5$ and the matrices

$$
\begin{aligned}
& W_{0}=\left(\begin{array}{cc}
1.0766 & -1.9225 \\
-1.9225 & 6.3512
\end{array}\right), W_{1}=\left(\begin{array}{cc}
0.5379 & -0.8637 \\
-0.8637 & 2.7929
\end{array}\right) \text {, } \\
& W_{2}=\left(\begin{array}{cc}
0.6935 & -1.8198 \\
-1.8198 & 5.3915
\end{array}\right), Z=\left(\begin{array}{cc}
0.8711 & -0.3431 \\
-0.3431 & 1.0586
\end{array}\right) \text {, }
\end{aligned}
$$

and following the methodology proposed in (Ochoa \& Kharitonov 2005, Garcia-Lozano \& Kharitonov 2006) we compute the function $U(\tau, W)$ associated to $W=W_{0}+W_{1}+h W_{2}-$ $Z A-A^{T} Z$. In this case, $u_{o z}=87.174$ and $u_{1}=88.0726$.

Third, using (29) we have $\gamma \in(0,0.002378)$. Substituting $\gamma=0.002377$ into (40) yields $\varepsilon=0.5554$. We also have from (56) and (57) that $\alpha_{1} \in(0,6.2347]$ and $\alpha_{2} \geq 153.1868$.

Fourth, we conclude from Theorem 7 that for $\alpha_{1}=6.2346$ and $\alpha_{2}=153.1868$ the set

$$
\Omega=\left\{\psi \in \mathfrak{C}:\|\psi\|_{0.5} \leq 0.05603\right\}
$$

is an estimate of the region of attraction of the trivial solution of system (4).

Finally, it follows from Corollary 8 that the solution $y(t, \psi)$ of system (44) such that $\psi \in \Omega$ satisfies

$$
\|y(t, \psi)\| \leq 5.394\|\psi\|_{0.5} e^{-2.593 \times 10^{-7} t}, \quad t \geq 0
$$

Summary of results for models I to IV

\begin{tabular}{|c|c||c|c|}
\hline \multirow{2}{*}{ Parameter } & \multicolumn{2}{|c||}{ Region of Attraction } & \multicolumn{2}{c|}{ Exponential estimate } \\
\cline { 2 - 4 } & $\nu$ & $\delta$ & $\varsigma$ \\
\hline Model I & 0.05603 & 5.394 & $2.593 \times 10^{-7}$ \\
\cline { 2 - 4 } Model II & 0.00618 & 5.4471 & $1.054 \times 10^{-7}$ \\
\cline { 2 - 4 } Model III & 0.00479 & 53.81 & $6.72 \times 10^{-7}$ \\
\cline { 2 - 4 } Model IV & 0.00846 & 18.5 & $3.67 \times 10^{-7}$ \\
\hline
\end{tabular}

Remark 9 The estimates of the regions of attractions presented here are conservative. Notice that the matrices $W_{i}, i=0,1,2$ and $Z$ can be used as free parameters for further improvements.

\section{$5 \quad$ Estimates of the region of attraction based on polar coordinates}

The estimates obtained in the previous section are very conservative due to the type of inequalities used to compute $\gamma$ and $\varepsilon$. In this section we achieve a better estimate by using 
a direct model analysis to find the constant $\rho$ greater than $\varepsilon / 2$ such that

$$
\|y(t)\|<\rho \Rightarrow \dot{v}\left(y_{t}\right)<0, t \geq 0 .
$$

As in the previous section, we only present the detailed analysis for Model I. The analysis of the other models is similar to the first one.

If the nominal system of (4) is exponentially stable, then there exists a unique LyapunovKrasovskii quadratic functional of complete type $v(\cdot)$ such that the time derivative of the functional along the trajectories of system (41) is

$$
\begin{aligned}
\dot{v}\left(y_{t}\right)= & -w\left(y_{t}\right) \\
& +2 f^{T}(y(t), y(t-h))[[U(0, W)-Z] y(t) \\
& \left.+\int_{-h}^{0} U(-h-\tau, W) A_{1} y(t+\tau) d \tau\right], \quad t \geq 0 .
\end{aligned}
$$

where the function $f(y(t), y(t-h))$ and the matrix $A_{1}$ are given by (6) and (5), respectively. Now, we define $U(0, W)-Z:=\left(\begin{array}{l}u_{01} u_{02} \\ u_{02} u_{03}\end{array}\right), U(-h-\tau, W):=\left(\begin{array}{l}u_{11}(-h-\tau) u_{12}(-h-\tau) \\ u_{21}(-h-\tau) u_{22}(-h-\tau)\end{array}\right)$. Substituting into (42) we have that

$$
\begin{aligned}
\dot{v}\left(y_{t}\right) & =-w\left(y_{t}\right) \\
& -2 \kappa_{4} \bar{F}_{p}\left[u_{02} y_{1}(t) y_{2}(t) y_{2}(t-h)+u_{03} y_{2}^{2}(t) y_{2}(t-h)\right] \\
& +2\left(\kappa_{4} \bar{F}_{p}\right)^{2}\left(P_{L}-\kappa_{5}\right) \int_{-h}^{0} u_{22}(-h-\tau) y_{2}(t) y_{2}(t-h) y_{2}(t+\tau) d \tau \\
& \leq-w\left(y_{t}\right) \\
& +2 \kappa_{4} \bar{F}_{p}\left[\left|u_{02}\right|\left|y_{1}(t) y_{2}(t) y_{2}(t-h)\right|+\left|u_{03}\right|\left|y_{2}^{2}(t) y_{2}(t-h)\right|\right] \\
& +2\left(\kappa_{4} \bar{F}_{p}\right)^{2}\left(P_{L}-\kappa_{5}\right) u_{22} \int_{-h}^{0}\left|y_{2}(t) y_{2}(t-h) y_{2}(t+\tau)\right| d \tau,
\end{aligned}
$$

where $u_{22}=\max _{\tau \in[-h, 0]}\left\{\left|u_{22}(-h-\tau)\right|\right\}$.

Taking

$$
y_{1}(t)=\rho(t) \sin \theta(t), \quad \text { and } \quad y_{2}(t)=\rho(t) \cos \theta(t),
$$

leads to

$$
\begin{aligned}
\|(y(t), y(t-h), y(t+\tau))\|^{2} & =\|y(t)\|^{2}+\|y(t-h)\|^{2}+\|y(t+\tau)\|^{2}, \tau \in[-h, 0] \\
& \leq 3 \rho^{2}, \tau \in[-h, 0],
\end{aligned}
$$


where $\rho=\max _{\tau \in[-h, 0]}\{\rho(t+\tau)\}$.

Now, substituting (45) and (44) into (43) we get

$$
\begin{aligned}
\dot{v}\left(y_{t}\right) \leq \int_{-h}^{0}\{ & -3 \lambda_{\min }(N) \rho^{2} \\
& +2 \kappa_{4} \bar{F}_{p} \rho^{3}\left[\frac{\left|u_{02}\right|}{h}|\sin \theta(t) \cos \theta(t) \cos \theta(t-h)|+\frac{\left|u_{03}\right|}{h}|\cos \theta(t) \cos \theta(t-h)|\right] \\
& \left.+2\left(\kappa_{4} \bar{F}_{p}\right)^{2}\left(P_{L}-\kappa_{5}\right) u_{22} \rho^{3}|\cos \theta(t) \cos \theta(t-h) \cos \theta(t+\tau)|\right\} d \tau
\end{aligned}
$$

where

$$
N=\left(\begin{array}{ccc}
W_{0} / h & Z A_{1} / h & 0 \\
A_{1}^{T} Z / h & W_{1} / h & 0 \\
0 & 0 & W_{2}
\end{array}\right) .
$$

We see that, $|\sin \theta(t) \cos \theta(t) \cos \theta(t-h)| \leq 0.5,|\cos \theta(t) \cos \theta(t-h)| \leq 1$, and $|\cos \theta(t) \cos \theta(t-h) \cos \theta(t+\tau)| \leq 1$, for all $t \geq 0$, therefore

$$
\dot{v}\left(y_{t}\right) \leq \int_{-h}^{0}\left(-3 \lambda_{\min }(N) \rho^{2}+2 \kappa_{4} \bar{F}_{p}\left[\frac{0.5\left|u_{02}\right|}{h}+\frac{\left|u_{03}\right|}{h}+u_{22} \kappa_{4}\left(P_{L}-\kappa_{5}\right) \bar{F}_{p}\right] \rho^{3}\right) d \tau .
$$

Thus,

$$
\text { if }\|y(t)\|<\rho, \quad \text { then } \dot{v}\left(y_{t}\right)<0, \quad, \quad \forall t \geq 0,
$$

where

$$
\rho=\frac{3 \lambda_{\min }(N)}{2 \kappa_{4} \bar{F}_{p}\left[\frac{0.5\left|u_{02}\right|}{h}+\frac{\left|u_{03}\right|}{h}+u_{22} \kappa_{4}\left(P_{L}-\kappa_{5}\right) \bar{F}_{p}\right]} .
$$

We are now ready to estimate the region of attraction of system (44) with the help of Theorem 7 .

Choosing $h=0.5$ and the matrices

$$
\begin{aligned}
& W_{0}=\left(\begin{array}{cc}
0.5 & 0 \\
0 & 0.5
\end{array}\right), W_{1}=\left(\begin{array}{cc}
0.5 & 0 \\
0 & 0.5
\end{array}\right) \\
& W_{2}=\left(\begin{array}{ll}
1 & 0 \\
0 & 1
\end{array}\right), \quad Z=\left(\begin{array}{ll}
0 & 0 \\
0 & 0
\end{array}\right) \text {, }
\end{aligned}
$$

we compute the function $U(\tau, W)$ associated to $W=W_{0}+W_{1}+h W_{2}-Z A-A^{T} Z$ (Ochoa \& Kharitonov 2005). For this case we obtain that $\left|u_{02}\right|=2.6,\left|u_{03}\right|=1.76$ and $u_{22}=1.76$. It follows from (47) that $\lambda_{\min }(N)=1$, using (49) we obtain $\rho=26.06877$. We also have from (56) and (57) that $\alpha_{1} \in(0,0.898]$ and $\alpha_{2} \geq 296.56$. 
Thus, for $\alpha_{1}=0.897$ and $\alpha_{2}=296.56$ it follows from Theorem 7 that the set

$$
\Omega=\left\{\psi \in \mathfrak{C}:\|\psi\|_{0.5} \leq 1.4341\right\}
$$

is an estimate of the region of attraction of the trivial solution of system (4).

Summary results for models I to IV

\begin{tabular}{|c|c|c|c|c|}
\hline & & & & Region of attraction \\
parameters & $\sigma$ & $\alpha_{1}$ & $\alpha_{2}$ & $\nu$ \\
\hline Model I & 26.068 & 0.897 & 296.56 & 1.4341 \\
Model II & 6.514 & 3.387 & 28.63 & 2.2407 \\
Model III & 0.489 & 0.02 & 89.23 & 0.00748 \\
Model IV & 1.016 & 0.0227 & 32.1 & 0.02702 \\
\hline
\end{tabular}

Remark 10 For the Model IV we use spherical coordinates:

$$
y_{1}(t)=\rho \sin \theta(t) \cos \phi(t), y_{2}(t)=\rho \sin \theta(t) \sin \phi(t), \text { and } y_{3}(t)=\rho \cos \theta(t),
$$

\section{Discussion of results}

In the following table we summarize relevant data from sections 4 and 5 . The first column is the $\mathrm{CO}_{2}$ arterial partial pressure in lung equilibrium, while the second and third columns are the values of the parameter $\nu$ for the estimates of the region of attraction obtained via the functional general approach and the polar coordinate direct approach, respectively.

\begin{tabular}{|c|c|c|c|}
\hline & $P C_{\mathrm{O}_{2}}(\mathrm{mmHg})$ & $\nu$ (section 4) & $\nu$ (section 5) \\
\hline Model I & 39.97 & 0.05603 & 1.4341 \\
Model II & 29.18 & 0.0615 & 2.2407 \\
Model III & 39.57 & 0.004792 & 0.008458 \\
Model IV & 39.41 & 0.008458 & 0.02702 \\
\hline
\end{tabular}

The main advantage of the approach based on Lyapunov-Krasovskii functionals introduced in section 3 is that it allows to estimate the region of attraction of a wide class of systems using a systematic methodology. In addition, it allows to obtain exponential estimates of the system response. A consequence of the generality of the method is the conservativeness of the results. 
The approach of section 5 is based on the use of polar (or spherical) coordinates combined with a direct, case by case, analysis of the negativity of the time derivative of the functional. As one can observe on the above Table, a significant reduction of the conservatism of the result obtained with this direct approach, when compared to the approach of section 3 . The improvement is the consequence of the direct analysis of each model and of the use of polar coordinates. However, this approach does not provide a general methodology. Moreover, it is restricted to two or tree variable systems.

It is acknowledged in the literature (Gaohua \& Kimura 2008), (Howard, Milhorn, Benton, Ross \& C. Guyton 1965) that the $\mathrm{CO}_{2}$ arterial partial pressures in lungs is about $40 \mathrm{mmHg}$. Clearly, the equilibriums of the model of Kollar (Kollár \& Turi 2004) is far from this value while those of Vielle, Batzel y Batzel II are close to it. As the model of Vielle provides the less conservative region of attraction and a realistic equilibrium we recommend this model for analyzing the behavior of the system with respect to initial conditions.

\section{Concluding remarks}

In this paper, we derive conditions under which the trivial solution of the nonlinear respiratory system models proposed in (Vielle \& Chavet 1998), (Kollár \& Turi 2004), (Batzel \& Tran 2000b), and (Batzel \& Tran 2000a) are asymptotically stable, and we propose two estimate of the region of attraction and an exponential estimate for the solutions of systems that starting in the estimate of the region of attraction. The approach considered in the paper is based on the use of Lyapunov-Krasovskii functionals of complete type with a cross term in the time derivative.

\section{References}

Batzel, J. \& Tran, H. (2000a), 'Stability analysis of the human respiratory control system. Part II', J. Math. Biol. 41:80-102.

Batzel, J. \& Tran, H. (2000b), 'Stability of the human respiratory control system. Part I', J. Math. Biol. 41:45-79.

Cooke, K. L. \& Turi, J. (1994), 'Stability, instability in delay equations modelling human respiration', Journal of Mathematical Biology 32, pp. 535-543.

Coutinho, D. F. \& de Souza, C. E. (2008), 'Delay-dependent robust stability and 12-gain 
analysis of a class of nonlinear time-delay systems', Automatica 44:2006-2008.

Gaohua, L. \& Kimura, H. (2008), 'A mathematical model of respiratory and biothermal dynamics in brain hypothermia treatment', IEEE Transactions on Biolmedical engineering 55(4):1266-1278.

Garcia-Lozano, H. \& Kharitonov, V. L. (2006), Numerical computation of time delay Lyapunov matrices, Proceedings of the 6th ifac workshop on time delay systems, L'Aquila, Italy.

Gu, K., Kharitonov, V. \& Chen, J. (2003), Stability of Time-Delay Systems, Control Engineering, Birkhäuser, USA.

Hoppensteadt, F. C. \& Peskin, C. S. (1992), Mathematics in Medicine and Life Sciences, TAM, vol. 10, Springer-Verlag: New York.

Howard, T., Milhorn, J., Benton, R., Ross, R. \& C. Guyton, A. (1965), 'A mathematical model of the human respiratory control system', Biophysical Journal 5:27-46.

Kharitonov, V. \& Zhabko, A. (2003), 'Lyapunov-Krasovskii approach to the robust stability analysis of time-delay system', Automatica 39:15-20.

Kollár, L. E. \& Turi, J. (2004), Numerical stability analysis in respiratory control system models, Conference on Diff. Eqns. and Appl. in Math. Biology 65-78, Nanaimo, BC, Canada.

Kolmanosvkii, V. \& Myshkis, A. (1999), Introduction to the theory and applications of functional differential equations, Kluwer Academyc Publisher.

Krasovskii, N. (1956), 'On the application of the second method of Lyapunov for equations with time delays', Prikl. Mat. Meh. 20:315-327.

Melchor-Aguilar, D. \& Niculescu, S.-I. (2007), 'Estimates of the attraction region for a class of nonlinear time-delay systems', IMA Journal on Mathematical Control and Information .

Mondié, S., Kharitonov, V. \& Santos, O. (2005), Complete type Lyapunov-Krasovskii functionals with a given cross term in the time derivative, Proceeding of the European Control Conference, Seville, Spain.

Murray, J. D. (1993), Mathematical Biology, BioMathematics Series, vol. 18, SpringerVerlag: Berlin. (second edition).

Ochoa, G. \& Kharitonov, V. (2005), 'Lyapunov matrices for neutral type time delay sys- 
tems', 2nd ICEEE 347-350.

Timischl, S. (1998), A global model for cardiovascular and respiratory system, PhD thesis, Karl-Franzens University of Graz, Austria.

Vielle, B. \& Chavet, G. (1998), 'Delay equation analysis of human respiratory stability', Mathematical Biosciences 105-122.

\section{Appendix}

In this appendix we recall the main results on the functionals of complete type needed in our analysis.

\subsection{Lyapunov-Krasovskii functionals of complete type}

We summarize the result presented in (Kharitonov \& Zhabko 2003) and (Mondié et al. 2005) on functionals with prescribed time derivative with cross terms.

We consider a time delay system of the form

$$
\dot{x}(t)=A_{0} x(t)+A_{1} x(t-h),
$$

where $A_{0}$ and $A_{1} \in \mathbb{R}^{n \times n}$.

Theorem 11 If the system (51) is exponentially stable, then for any given positive definite matrices $W_{i} \in \mathbb{R}^{n \times n}, i=0,1,2$ and a symmetric real matrix $Z \in \mathbb{R}^{n \times n}$ such that

$$
\left(\begin{array}{cc}
W_{0} & Z A_{1} \\
A_{1} Z & W_{1}
\end{array}\right)>0,
$$

the functional

$$
\begin{aligned}
v\left(x_{t}\right)= & -x^{T}(t) Z x(t)+x^{T}(t) U(0, W) x(t) \\
& +2 x^{T}(t) \int_{-h}^{0} U(-h-\tau, W) A_{1} x(t+\tau) d \tau \\
& +\int_{-h}^{0} \int_{-h}^{0} x^{T}\left(t+\tau_{1}\right) A_{1}^{T} U\left(\tau_{1}-\tau_{2}, W\right) A_{1} x\left(t+\tau_{2}\right) d \tau_{1} d \tau_{2} \\
& +\int_{-h}^{0} x^{T}(t+\tau)\left[W_{1}+[h+\tau] W_{2}\right] x(t+\tau) d \tau,
\end{aligned}
$$

satisfies the following:

$$
\dot{v}\left(x_{t}\right)=-w\left(x_{t}\right), \quad \forall t \geq 0
$$


and

$$
\alpha_{1}\|x(t)\|^{2} \leq v\left(x_{t}\right) \leq \alpha_{2}\left\|x_{t}\right\|_{h}^{2}, \quad \forall t \geq 0,
$$

where

$$
\begin{aligned}
w\left(x_{t}\right)= & x^{T}(t) W_{0} x(t)+x^{T}(t-h) W_{1} x(t-h) \\
& +2 x^{T}(t) Z A_{1} x(t-h) \\
& +\int_{-h}^{0} x^{T}(t+\tau) W_{2} x(t+\tau) d \tau .
\end{aligned}
$$

$\alpha_{1} \in\left(0, \alpha^{*}\right]$, with $\alpha^{*}$ such that

$$
\left(\begin{array}{cc}
W_{0} & Z A_{1} \\
A_{1}^{T} Z & W_{1}
\end{array}\right)+\alpha^{*}\left(\begin{array}{ccc}
A_{0}+A_{0}^{T} & A_{1} \\
A_{1}^{T} & 0
\end{array}\right)>0,
$$

and $\alpha_{2}>0$ satisfies

$$
\alpha_{2} \geq \kappa(1+h)
$$

Here, $\kappa \geq \max \left\{u_{z o}+h u_{1} a_{1}, u_{1} a_{1}+w_{1}+h w_{2}+h u_{1} a_{1}^{2}\right\}, u_{o z}=\|U(0, W)-Z\|, u_{1}=$ $\max _{\tau \in[0, h]}\{\|U(\tau, W)\|\}, a_{1}=\left\|A_{1}\right\|$ and $w_{i}=\left\|W_{i}\right\|, i=1,2$.

Remark 12 The matrix $U(\tau, W)$ is called the Lyapunov matrix associated to $W=W_{0}+$ $W_{1}+h W_{2}-A_{0}^{T} Z-Z A_{0} \in \mathbb{R}^{n \times n}$ and the unique solution of the analogue of the Lyapunov Equation for time delay systems is (Ochoa 83 Kharitonov 2005)

$$
\begin{aligned}
& U^{\prime}(\tau, W)=U(\tau, W) A_{0}+U(\tau-h, W) A_{1}, \quad \tau \geq 0, \\
& U(-\tau, W)=U^{T}(\tau, W), \quad \tau \geq 0, \\
& -W=U^{\prime}(+0, W)-U^{\prime}(-0, W) .
\end{aligned}
$$

